INTERVENTIONAL CARDIOLOGY AND SURGERY

\title{
Long term follow up after elective percutaneous coronary intervention for unprotected non-bifurcational left main stenosis: is it time to change the guidelines?
}

\author{
B R G Brueren, J M P G Ernst, M J Suttorp, J M ten Berg, B J W M Rensing, E G Mast, E T Bal, A J Six, \\ H W M Plokker
}

Heart 2003;89:1336-1339

Background: According to the American College of Cardiology/American Heart Association guidelines, percutaneous coronary intervention (PCI) for left main coronary artery (LMCA) stenosis is contraindicated and coronary artery bypass graft surgery (CABG) is preferred. However, $\mathrm{PCl}$ of the LMCA is performed under exceptional circumstances.

Objective: To analyse the data of patients who underwent PCl of the unprotected LMCA in St Antonius Hospital, Nieuwegein, Netherlands.

See end of article for authors' affiliations Results: In a database of $17683 \mathrm{PCl}$ procedures, 71 patients $(0.4 \%)$ were found with non-bifurcational LMCA stenosis who underwent an elective PCl between 1991 and 2001. Ages ranged from 26.7-86.5 years. Severe concomitant disease was the most frequent argument in favour of $\mathrm{PCl}$ instead of CABG. PCI Correspondence to: Dr B R G Brueren, Heart Lung Centre Utrecht, St Antonius Ziekenhuis, Koekoekslaan 1, 3435 CM Nieuwegein, The Netherlands; brg_brueren@ hotmail.com

Accepted 9 April 2003 consisted of only balloon angioplasty in 23 cases (32.4\%). A stent was used in 46 cases (64.4\%). Average follow up was 43 months (range 0-121 months). One patient died one day after the procedure. The total one year survival rate was $98.6 \%(70 / 71)$. Seven patients died during the follow up period, mostly because of non-cardiac reasons. The annual mortality rate was $2.5 \%$. Recurrent elective percutaneous transluminal coronary angioplasty for restenosis of the LMCA was performed in one patient (1.4\%) six weeks after the initial procedure. CABG was required in 13 patients $(18.3 \%)$ throughout the follow up period.

Conclusion: These results suggest that at highly experienced centres, elective $\mathrm{PCl}$ of the non-bifurcational LMCA can be performed safely where the anatomy is suitable.

S ince its introduction in $1977^{1}$ percutaneous coronary intervention (PCI) has been applied increasingly and today most types of atherosclerotic coronary artery disease can be treated percutaneously. One of the few contraindications remaining is stenosis of the left main coronary artery (LMCA). This potential indication is considered to be too dangerous because of the expected high mortality of acute closure, in contrast with coronary artery bypass graft surgery (CABG), which can be performed with a risk $<5 \%{ }^{2}$ Therefore, LMCA stenosis has been excluded in most trials and should be avoided according to the American College of Cardiology (ACC)/American Heart Association (AHA) guidelines. ${ }^{3}$ However, PCI of the LMCA has been practised under special circumstances, such as under "protection" by means of a bypass graft, in the setting of acute myocardial infarction or in case of acute closure caused by catheter manipulation during diagnostic catheterisation. Because of the experience with PCI of the LMCA under special circumstances this technique is applied increasingly in broader categories of patients. Our study challenges the discouraging comments in the current guidelines.

In the setting of continuous evaluation of the quality of care, we analysed the data of patients who underwent PCI of the unprotected LMCA in our hospital.

\section{METHODS}

Patient selection

Between January 1990 and July 2001, 17683 PCI procedures were performed at our site. A database of relevant patient characteristics and procedural data was maintained. PCI of the LMCA was performed in 218 patients $(1.2 \%)$. In 104 patients $(0.6 \%)$ this involved PCI of the unprotected LMCA. Twenty four of these patients $(23.1 \%)$ were excluded for this study because of the setting of acute myocardial infarction. Nine other patients $(8.7 \%)$ were excluded, as this procedure involved an emergency treatment resulting from dissection during coronary angiography. Consequently, this analysis involves 71 patients with elective PCI of the unprotected LMCA, representing only $0.4 \%$ of the total PCI population.

Only stenosis proximal to the bifurcation was considered suitable for catheter interventions. In contrast, we always considered PCI in a short LMCA and with stenosis involving the bifurcation to be contraindicated.

\section{Definition of end points}

Mortality was analysed based on actuarial data. One year follow up was obtained in all but one patient. Acute myocardial infarction was defined as clinical documentation including typical electrocardiographic changes or increased cardiac enzyme concentrations. Repeated PCI was defined as any catheter based intervention performed more than one hour after the index procedure. Left ventricular function was classified semiquantitatively based on pretreatment left ventricular angiography.

Abbreviations: ACC, American College of Cardiology; AHA American Heart Association; $C A B G$, coronary artery bypass graft surgery; LMCA, left main coronary artery; $\mathrm{PCl}$, percutaneous coronary intervention; PTCA, percutaneous transluminal coronary angioplasty 


\section{Statistical analysis}

Data are presented as percentage incidence, mean (SD), or median and interquartile range as appropriate. Total and event-free survival were determined using Kaplan-Meier statistics.

\section{RESULTS}

\section{Patients}

Table 1 gives the patients' characteristics. The age of the patients ranged from 26.7-86.5 years at the time of procedure. Ten patients $(14.1 \%)$ had a previous myocardial infarction, 30 patients $(42.3 \%)$ had a normal ECG, and six patients $(8.5 \%)$ had undergone a PCI previously. Table 2 presents angiographic data. In 35 (49.3\%) cases, the patient had LMCA stenosis only, while the other 36 (50.7\%) had an additional significant stenosis in one or more of the branches of the coronary tree. The average stenosis diameter as assessed by quantitative coronary angiography was $69 \%$. Twenty one patients $(29.6 \%)$ had only minimal calcifications. In six patients $(8.5 \%)$ excellent collaterals to the perfusion area of the LMCA were noted. Left ventricular function was normal in 43 patients $(60.6 \%)$, slightly diminished in 13 patients $(18.3 \%)$, and moderately to severely impaired in 12 patients $(16.9 \%)$. In three $(4.2 \%)$ patients data on left ventricular function were not available.

\section{Procedure}

PCI consisted of balloon angioplasty only in 23 cases (32.4\%). A stent was used in 46 cases $(64.4 \%)$. In one case a cutting balloon was used. In one other case a rotablator was applied. An intra-aortic balloon pump was not routinely used.

\section{Indications}

Severe concomitant disease was an argument for choosing PCI instead of CABG in $37(51.1 \%)$ patients. Very severe peripheral arterial occlusive disease was reported in 19 $(26.8 \%)$ patients, severe chronic obstructive pulmonary disease (with reduced pulmonary capacity) in six $(8.5 \%)$, cardiomyopathy in four $(5.6 \%)$, recent myocardial infarction in four $(5.6 \%)$, severe renal insufficiency in three $(4.2 \%)$, and malignant tumours in one (1.4\%). In $34(47.9 \%)$ patients the decision to perform PCI was based on the combination of patient preference and feasible anatomy, without a clear medical contraindication for CABG. In all cases the increased risk of the procedure was carefully considered, discussed with the referring cardiologist, and explained clearly to the patient, who was free to choose any possible alternative.

\section{Medical treatment}

All but one of the patients were taking aspirin. All patients received heparin during the procedure. All stent patients were given either ticlopidine or clopidogrel after stenting, and 21 patients were treated with oral anticoagulants.

Table 1 Patient characteristics

\begin{tabular}{ll}
\hline Characteristic & Number of patients \\
\hline Total PCI & 17683 \\
LMCA PCI & $218(1.2 \%)$ \\
Unprotected, elective LMCA PCI & $71(0.4 \%)$ \\
Mean (SD) age (years) & $60.4(12.6)$ \\
Male sex & $43(60.1 \%)$ \\
Risk factors & \\
Smoking & $22(31.0 \%)$ \\
Known hypercholesterolaemia & $28(39.4 \%)$ \\
Diabetes & $5(7.0 \%)$ \\
Family history of CAD & $21(29.6 \%)$ \\
\hline
\end{tabular}

$C A D$, coronary artery disease; $L M C A$, left main coronary artery; $\mathrm{PCl}$, percutaneous coronary intervention.

\begin{tabular}{ll} 
Table 2 & Baseline angiography \\
\hline Result & Number of patients \\
\hline Coronary stenosis & \\
LMCA only & $35(49.3 \%)$ \\
LMCA plus: & $10(14.1 \%)$ \\
1 vessel & $24(33.8 \%)$ \\
2 vessel & $2(2.8 \%)$ \\
3 vessel & $21(29.6 \%)$ \\
Calcification & $6(8.5 \%)$ \\
Collaterals &
\end{tabular}

\section{Procedural data}

During the procedure dissection of the LMCA occurred in 11 patients (table 3 ). In one patient (1.4\%) urgent CABG was required immediately upon PCI because a dissection spread out into the left anterior descending and right circumflex arteries. The surgical procedure was performed and the clinical course was without sequelae.

Urgent repeat angiography within 48 hours was necessary in two cases. In one case a repeat balloon angioplasty only within 30 minutes was needed because of acute closure and a bailout stent was placed. Acute myocardial infarction did not occur. This patient underwent elective CABG 10 months later. In the other case, acute lateral ischaemia occurred within one hour after the procedure. At angiography, the stent that was placed during the index procedure appeared to be patent. However, thrombus formation was seen in the circumflex branch. Dilatation was performed successfully. Cardiac enzyme concentrations did not rise.

\section{Follow up}

Average follow up was 43 months, ranging from 0-121 months. One patient died one day after the procedure, in whom a Medtronic AVE 3.5-16 mm stent (Medtronic AVE, Santa Rosa, California, USA) was used. In this patient, only heparin and aspirin had been given as concomitant antithrombotic medication. At that time (1993) clopidogrel and ticlopidine were not yet commercially available. Urgent coronary angiography showed massive thrombus formation in the left coronary artery. Redilatation was not technically possible. This patient died in cardiac shock during the second procedure. During the one year follow up period one other patient died of pulmonary malignancy 11 months after the PCI. The total one year survival was 70/72 (97.2\%).

Figure 1 shows Kaplan-Meier survival curves. Seven patients died during the follow up period. The annual mortality rate was $2.5 \%$. Most of these patients (5) died of non-cardiac natural causes: cancer (2), renal insufficiency (1), and cerebrovascular accident (2). One other patient died after a car accident. The single cardiac death, occurring periprocedurally, is described above.

Figure 2 shows event-free survival. After one year, 97.2\% of the patients were alive and free of both repeat percutaneous transluminal coronary angioplasty (PTCA) and CABG. Recurrent elective PTCA for restenosis of the LMCA was

Table 3 Procedural complications

\begin{tabular}{ll}
\hline Complication & Number of patients \\
\hline Dissection & $11(15.3 \%)$ \\
IABP placed & 0 \\
Resuscitation & 0 \\
Urgent CABG & $1(1.4 \%)$ \\
Urgent angiography & $2(2.8 \%)$ \\
Periprocedural death & $1(1.4 \%)$ \\
\hline
\end{tabular}

$C A B G$, coronary artery bypass grafting; IABP, intra-atrial balloon pump. 


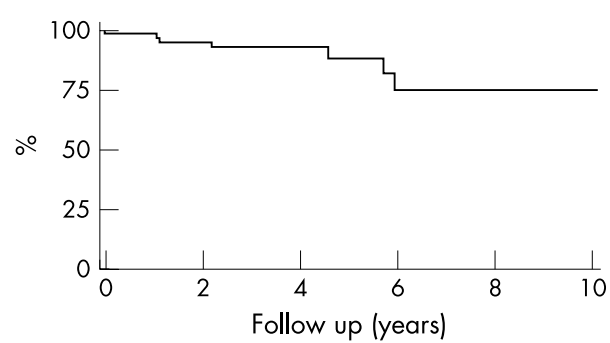

Figure 1 Kaplan-Meier survival curve: all cause mortality.

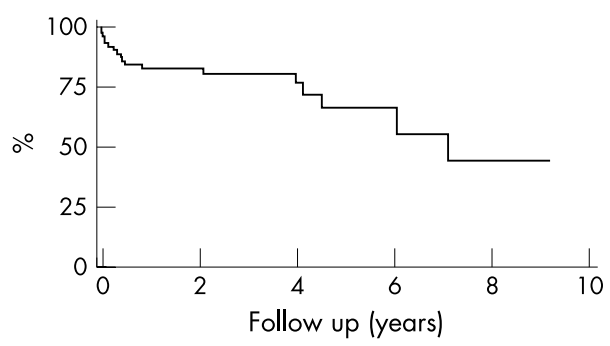

Figure 2 Kaplan-Meier survival curve for patients free from coronary artery bypass graft surgery (CABG) and any repeat percutaneous coronary intervention.

performed in one patient six weeks after the initial procedure. CABG was required in 13 patients throughout the follow up period, in one case because of restenosis and in 12 cases because of progression of stenosis elsewhere. Figure 3 shows survival free from CABG. There was one periprocedural myocardial infarction, which is described above.

\section{DISCUSSION}

PCI of the unprotected LMCA has been discouraged by the ACC/AHA. ${ }^{3}$ Therefore, there is only limited experience with elective PCI of unprotected LMCA stenosis, reflected by only 10 publications on studies involving more than 10 patients. ${ }^{4-13}$ These studies report an average periprocedural mortality of $8.8 \%$ (range $0-36 \%$ ) in a total of 799 patients. Thus, it is clear that PCI of a significant stenosis of the LMCA carries significant risks and strongly reduced short term survival. The three year mortality rate in case of LMCA stenosis treated only with drugs may be at least $50 \%$ according to various publications from the 1970s. ${ }^{14-17}$ Acute closure of this vessel may result in immediate death, while bypass surgery in these patients can be performed without significant risk. ${ }^{18} 19$

Initial experience in the 1980s with PCI for LMCA stenosis was indeed quite discouraging. O'Keefe et a ${ }^{20}$ reported a $9.1 \%$ procedural death rate in 1989. In the early series published by Tomasso et al, ${ }^{4}$ Keeley et al, ${ }^{5}$ and Chauhan et al in-hospital mortality rates of $14 \%, 5 \%$, and $36 \%$, respectively, were reported. These rates have gradually decreased to $0-13.7 \%$ in more recent studies. ${ }^{7-13}$ The well documented registry by Silvestri et $a l^{7}$ reports a relatively favourable short term outcome in terms of an in-hospital mortality of 3\% in 140 patients treated between 1993 and 1998 at a single site. However, in this series the one year mortality was as high as $13 \%$. Ellis et $a l^{8}$ reported unfavourable early results as shown by an in-hospital mortality of $12 \%$ in 91 patients, and by a six month mortality risk of $30 \%$. The somewhat smaller studies by Park et $a l^{9}$ and Wong et a $l^{10}$ found $100 \%$ early survival, but long term figures are lacking. The recently published multicentre registry of 279 patients by Tan et $a l^{13}$ had an in-hospital mortality of $13.7 \%$ and of $26.8 \%$ at one year.

In fact, the average early mortality of $8.8 \%$ in the 10 recently published major studies mentioned in the introduction of this paper truly is a strong argument in favour of

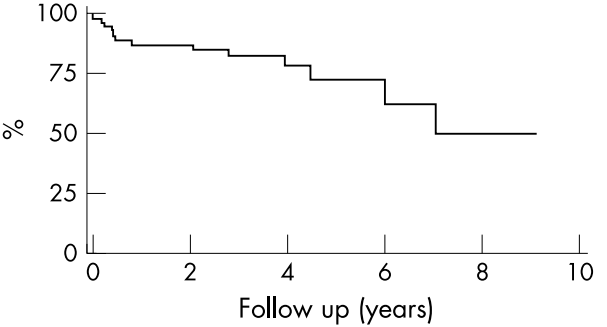

Figure 3 Kaplan-Meier survival curve: CABG-free cardiac survival.

CABG in patients with LMCA stenosis. Only in the case of severe concomitant disease may the risk to benefit ratio favour angioplasty, when the risk of bypass surgery is considered unacceptably high. Also in our hospital the number of patients who underwent PCI of LMCA is very low $(0.4 \%)$. Initially, we treated only patients with severe co-morbidity, which was present in $52(73.2 \%)$ patients of the study population. As the safety of the procedure has increased with improvement of procedural techniques, drugs, and experience of the operators, we have gradually come to regard PCI of LMCA as a viable option. Although it is not routinely presented as an attractive alternative to CABG, sometimes patients put pressure on the treating cardiologist to make a case for PCI. The policy at our site remains not to encourage this treatment. However, when the patient is well informed and prepared to make his or her own decision based on risk stratification, we are willing to comply. We stress again that in the given time period unprotected LMCA stenosis was an extremely rare indication for PCI, occurring in only $0.4 \%$ of the total PCI population.

The in-hospital mortality was $1.4 \%$. We were pleasantly surprised to find that the long term follow up results in our patients, both those treated without stents in the early days and those treated with stents, were excellent. Our long term Kaplan-Meier analyses show event rates that may be comparable with the results of CABG in patients with severe coronary artery disease. A significant mortality is seen after approximately 4-6 years, as fig 1 shows. All three patients died of end stage conditions of concomitant diseases, such as metastasised cancer or renal insufficiency. Meanwhile, these patients obtained significant medical benefit from the cardiac intervention, avoiding severe angina pectoris and myocardial infarction. It should be stressed that concomitant disease, which typically is excluded from randomised trials, was the reason for the unusual interventional approach. In the literature there is only limited information on long term follow up. Only Keeley et al provide more than two years of follow up data. However, that study combined elective, emergent, protected, and unprotected cases. Detailed information on the subgroups is not always provided. Therefore, that study does not provide a clear view of the prognosis of patients undergoing elective PCI of the unprotected LMCA.

The favourable results at our site require further explanation. We believe that, in general, the methods applied at the Antonius Hospital do not differ essentially from those applied at other large institutions in the USA or the European community. One explanation is the angiographic selection criteria: we perform this procedure only in non-bifurcational lesions in relatively long LMCA and not in the case of a short LMCA or atherosclerotic stenosis involving the bifurcation. We believe that in particular LMCA bifurcation lesions carry an unacceptably high risk. This is explained by the general opinion that, even in the area of stenting, bifurcational lesions have a particularly high recurrence rate. The relatively good results in our study group may also be explained in part by potentially higher success rates in more experienced 
hands, illustrated by an annual volume of more than 500 PCIs per interventional cardiologist at our site. This finding in PCI in general may hold true in particular for technically difficult or risky procedures such as PCI of the LMCA. ${ }^{20}$ In light of the study period, 1990-2001, it is not surprising that the number of stents used $(64.4 \%)$ is relatively low. In today's practice basically all patients would receive a stent. The advantages and disadvantages of drug eluting stents are under study. It is not a policy at our site to routinely perform long term control angiography. We believe in non-invasive assessments of coronary ischaemia.

Our study did not include any kind of control group. A prospective randomised comparison would be desirable. A comparison with drug treatment only would be unethical because of the very poor prognosis of medically treated LMCA stenosis. Randomisation versus CABG could be a demanding but challenging option. Because of the selection criteria (nonbifurcational) it would take a very long time to recruit a large number of patients. However, what would be the benefit of surgery compared with a procedural risk of $1.4 \%$ and a coronary artery disease related annual mortality of just $1 \%$ ? Moreover, a considerable number of patients probably would prefer to undergo a PTCA procedure when asked to give consent in a prospective randomised trial.

\section{Conclusion}

We think that it is time for the ACC/AHA task force to reconsider its recommendations for PCI in patients with LMCA stenosis. Also, our patient information may be more optimistic about the risks of a PCI procedure for LMCA disease, again with emphasis on the anatomy. On the basis of our results retrieved from historical data, elective PCI of the non-bifurcational LMCA should not be principally avoided, at least in highly experienced sites and in the case of suitable lesion anatomy.

\section{Authors' affiliations \\ B R G Brueren, J M P G Ernst, M J Suttorp, J M ten Berg, B JW M Rensing, \\ E G Mast, E T Bal, A J Six, H W M Plokker, Department of Cardiology,}

St Antonius Hospital, Nieuwegein, The Netherlands

\section{REFERENCES}

1 Gruentzig AR. Transluminal dilatation of coronary artery stenosis. Lancet 1978;i:263.
2 d'Allonnes FR, Corbineau $\mathrm{H}$, Le Breton $\mathrm{H}$, et al. Isolated left main coronary artery stenosis: long term follow-up in 106 patients after surgery. Heart 2002;87:544-8.

3 Smith SC Jr, Dove JT, Jacobs AK, et al. ACC/AHA guidelines of percutaneous coronary interventions (revision of the 1993 PTCA guidelines) - executive summary. A report of the American College of Cardiology/American Heart Association task force on practice guidelines (committee to revise the 1993 guidelines for percutaneous transluminal coronary angioplasty). J Am Coll Cardiol 2001;37:2215-39.

4 Tommasso CL, Vogel JHK, Vogel RA for the national registry of elective supported angioplasty. Coronary angioplasty in high-risk patients with left main coronary stenosis: results from the national registry of elective supported angioplasty. Cathet Cardiovasc Diagn 1992;25:169-73.

5 Keeley EC, Aliabadi D, O'Neill WW, et al. Immediate and long-term results of elective and emergent percutaneous interventions on protected and unprotected severely narrowed left main coronary arteries. Am J Cardiol 1999;83:242-6.

6 Chauhan A, Zubaid M, Ricci DR, et al. Left main intervention revisited: early and late outcome of PTCA and stenting. Cathet Cardiovasc Diagn 1997;41:21-9

7 Silvestri M, Barragan P, Sainsous J, et al. Unprotected left main coronary artery stenting: immediate and medium term outcomes of 140 elective procedures. J Am Coll Cardiol 2000;35:1543-50.

8 Ellis SG, Tamai H, Nobuyoshi M, et al. Contemporary percutaneous treatment of unprotected left main coronary stenoses: initial results from a multi center registry analysis 1994-1996. Circulation 1997;96:3867-72.

9 Park S-J, Park S-W, Hong M-K, et al. Stenting of unprotected left main coronary stenoses: immediate and late outcomes. J Am Coll Cardiol 1998;31:37-42.

10 Wong $\mathbf{P}$, Wong $\mathrm{V}$, Tse $\mathrm{K}-\mathrm{K}$, et al. A prospective study of elective stenting in unprotected left main coronary disease. Cathet Cardiovasc Intervent 1999:46:153-9.

11 Laruelle CJJ, Brueren GBR, Ernst SMPG, et al. Stenting of "unprotected" left main coronary artery stenoses: early and late results. Heart 1998;79: 148-52.

12 Karam C, Fajadet J, Cassagneau B, et al. Results of stenting of unprotected left main coronary stenosis in patients at high surgical risk. Am J Cardiol 1998;82:975-8.

13 Tan WA, Tamai H, Park SJ, ef al for the ULTIMA Investigators. Long-term clinical outcomes after unprotected left main trunk percutaneous revascularization in 279 patients. Circulation 2001;104:1609-14.

14 Bruschke AVG, Proudit WL, Sones FM, et al. Progress study of 590 consecutive nonsurgical cases of coronary disease followed 5 to 9 years. Circulation 1973;47:1147-53.

15 Lim JS, Proudit WL, Sones FM, et al. Left main coronary arterial obstruction: long-term follow-up of 141 nonsurgical cases. Am J Cardiol 1975:36:131-5.

16 Cohen MV, Gorlin R. Main left coronary artery disease: clinical experience from 1964 to 1974. Circulation 1975;52:275-85.

17 Conley MJ, Ely RL, Kisslo J, et al. The prognostic spectrum of left main stenosis. Circulation 1978:57:947-52.

18 Varnanskas E, for the European Coronary Surgery Study Group. Twelve year follow-up of survival in the randomized European coronary surgery study. N Engl J Med 1988;319:332-7.

19 Caracciolo EA, Davis KB, Sopko G, et al. Comparison of surgical and medical group survival in patients with left main coronary artery disease. Long-term CASS experience. Circulation 1995;91:2325-34.

20 O'Keefe JH, Hartsler GO, Rutherford BD, et al. Left main coronary angioplasty: early and late results of 127 acute and elective procedures. Am J Cardiol 1989:64:144-7.

\section{www.heartjnl.com}

